\title{
Design issues for a Dexter-based hypermedia system
}

\author{
Kaj Grønbæk \\ Computer Science Department, Aarhus University, \\ Ny Munkegade 116, DK 8000 Aarhus C, Denmark. \\ Randall H. Trigg \\ Xerox PARC \\ 3333 Coyote Hill Rd., Palo Alto, CA 94304, USA
}

October 29, 1993

To appear in Communications of the ACM, February, 1994.

\begin{abstract}
This paper discusses experiences and lessons learned from the design of an open hypermedia system, one that integrates applications and data not "owned" by the hypermedia. The Dexter Hypertext Reference Model [8] was used as the basis for the design. Though our experiences were generally positive, we found the model constraining in certain ways and underdeveloped in others. For instance, Dexter argues against dangling links, but we found several situations where permitting and supporting dangling links was advisable. In Dexter, the data objects making up a component's contents are encapsulated in the component; in practice, references to objects stored apart from the hypermedia structure should be allowed. We elaborate Dexter's notion of composite component to include composites that "contain" other components and composites with structured contents, among others. The paper also includes a critique of Dexter's notion of link directionality, proposes a distinction between marked and unmarked anchors, and discusses anchoring within a composite.
\end{abstract}

\section{Introduction}

The hypermedia work discussed here is part of the DeVise project at the Computer Science Department, Aarhus University, Denmark [4]. The DeVise project is developing general tools to support experimental system development and cooperative design in a variety of application areas including large engineering projects. These use settings are characterized by group work distributed over time, space and hardware platforms. The requirements this intensely collaborative, open-ended work makes on hypermedia include: a shared database, access from multiple platforms, portability, extensibility and tailorability. For a detailed discussion of our engineering project use setting and its CSCW and hypermedia requirements, see [5].

To our knowledge, no hypermedia system met these requirements on the platforms we needed to support. Having to build our own, we nonetheless wanted to benefit from the experience and expertise of past and present hypermedia designers. It was for this reason that we decided to use the Dexter Hypertext Reference Model [8] (called "Dexter" in the rest of this paper) as our platform. Dexter is an attempt to capture the best design ideas from a group of "classic" hypertext systems, in a single overarching data and process model. Although these systems have differing design goals and address a variety of application areas, Dexter managed to combine and generalize many of their best features.

We took the Dexter reference model as the starting point and turned it into an object-oriented design and prototype implementation (called DeVise Hypermedia, or just "DHM"), running on 
the Apple Macintosh. As programming environment, we chose the Mjølner Beta System supporting the object oriented programming language BETA [12]. The Mjølner Beta System includes an object-oriented database [10], in which our hypermedia structures are stored.

Among the media supported by DHM are text, graphics, and video, using a styled text editor, a simple drawing editor, and a Quicktime movie player, respectively. DHM also supports link and node browser composites and a composite to capture screen configurations of open components (modeled on the NoteCards TableTop [18]). In addition to traversing links (including multi-headed ones), users can edit link endpoints using a graphical interface. Components can also be retrieved and presented via title search. Dexter's model of anchoring is extended to include a distinction between marked and unmarked anchors. Finally, in contrast to Dexter, DHM explicitly supports dangling links.

In short, our attempt to directly "implement" Dexter was largely successful. We were surprised at the robustness of the resulting design - it met several of our goals not explicitly identified in the Dexter paper. At the same time, we uncovered holes in the model, areas where further development is needed. For some of these, we now feel prepared to offer proposals for other hypermedia designers. Those involving the overall architecture, details of the data and process model, and tailorability are described in [3]

This paper reviews the Dexter model before discussing our experiences in applying it. Our focus here is on links, anchors, composites and cross-layer interfaces. For each of these, we comment on the utility and applicability of Dexter, identify the implementation choices made in our prototype, and make recommendations for designers of future systems and standards. We close with research issues and open questions.

\section{The Dexter Model}

The Dexter Hypertext Reference Model [8] separates a hypertext system into three layers having well defined interfaces as shown in Figure 1.

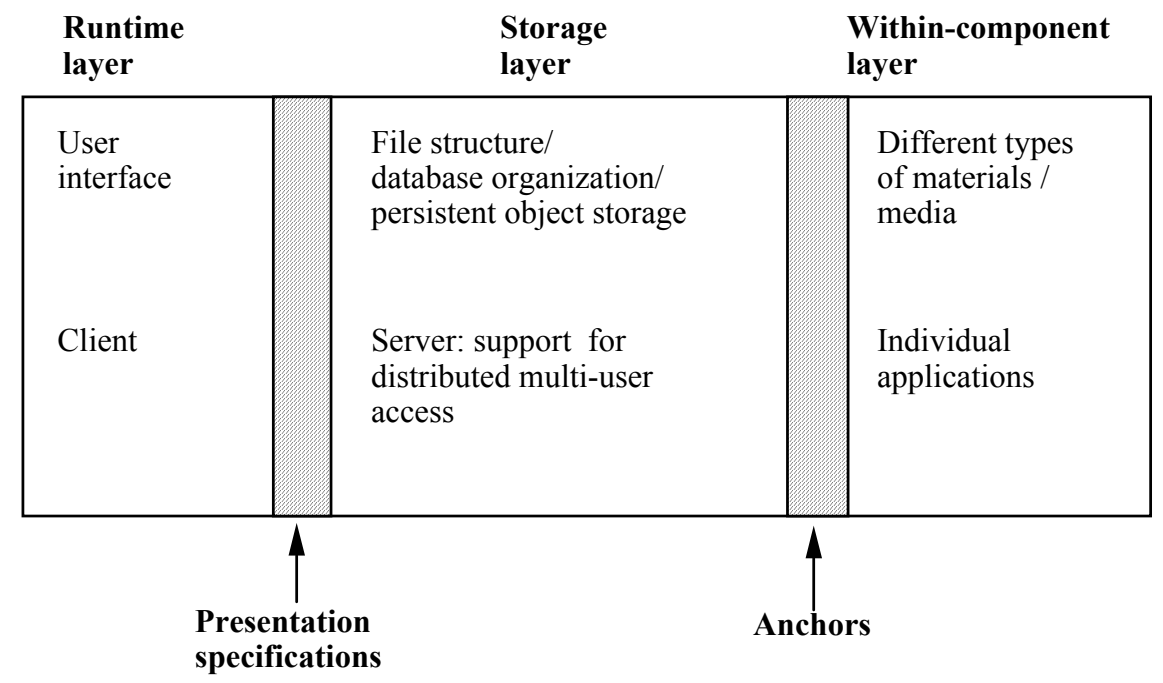

Figure 1: The Dexter model layers and interfaces. ${ }^{1}$

The Storage layer captures the persistent, storable objects making up the hypertext. The basic object provided in the Storage layer is the component. As shown in Figure 2, components are

\footnotetext{
${ }^{1}$ Some of the text appearing in the figure is our own.
} 
divided into contents, corresponding to the component's data, and component information. The component information includes a general purpose set of attributes, a presentation specification and a set of anchors. The atomic component is an abstraction replacing the widely used but weakly defined concept of 'nodes' in a hypertext network. Composite components provide a hierarchical structuring mechanism. The contents of a link component is a list of specifiers, each including a presentation specification as well as component and anchor identifiers. A hypertext is simply a set of components.
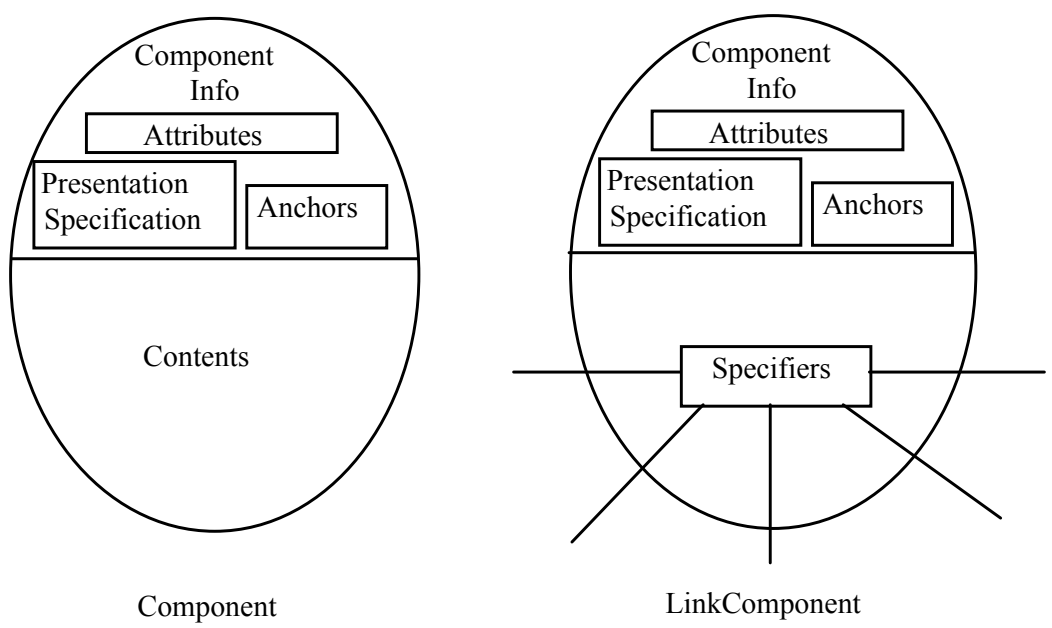

Figure 2: Component structure in the Storage Layer.

The Within-component layer corresponds to individual applications. The applications are responsible, for example, for supporting content selections for link anchoring.

The interface between the storage and within-component layers is based on the notion of anchors. Anchors consist of an identifier that can be referred to by links and a value that picks out the anchored part of the material.

The Runtime layer is responsible for handling links, anchors, and components at runtime. Objects in the runtime layer include sessions, managing interaction with particular hypertexts, and instantiations, managing interaction with particular components. The runtime layer provides tool independent user interface facilities through operations like NewComponent, AddLinkEndpoints, and FollowLink.

The interface between the Storage layer and the Runtime layer includes presentation specifications which determine how components are presented at runtime. Presentation specifications might include information on screen location and size of a presentation window, as well as a "mode" for presenting a component. Halasz and Schwartz [8] use the example of an animation component that can be opened in either run mode or edit mode.

\section{Links}

Links have traditionally formed the heart of hypertext systems. Indeed, the traversable network structures formed by links distinguish hypertext from other means of organizing information. Hypertext systems have implemented links in several ways, many of which are unified by Dexter's notion of link component. In addition to the typical source/destination links, Dexter can model multi-headed links. Furthermore, because links are components, they can be the endpoints of other links. Through the use of specifiers, the Dexter model supports computed as well as static links. Simple "typing" can be supported by adding attributes to the link component. But 
DHM also supports full-fledged typing of links due to its object oriented component design.

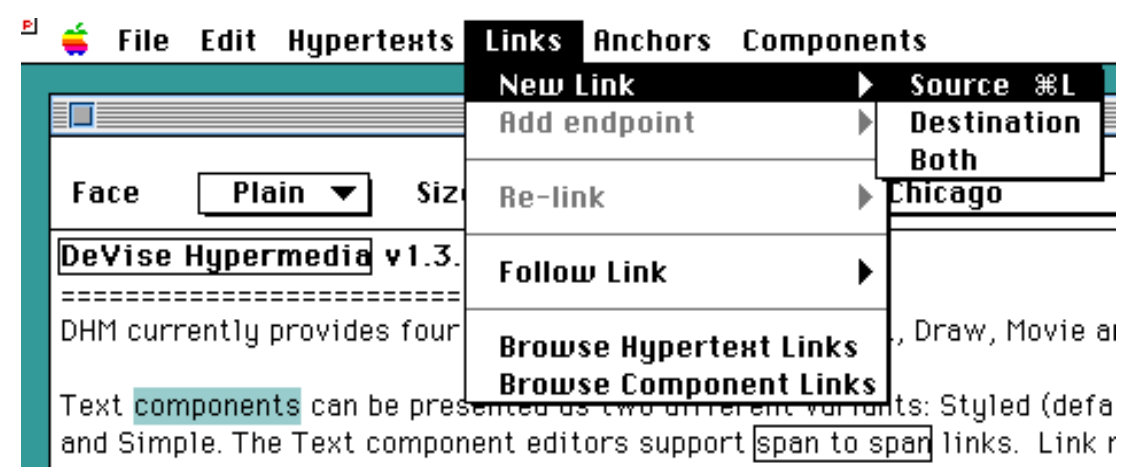

Figure 3: Creating a link with one source endpoint, anchored in the currently selected text: "components".

Though in principle a Dexter link could have fewer than two endpoints, this is expressly forbidden by the model's semantics. In DHM, we have relaxed this constraint; that is, "dangling" links having zero or one endpoint are perfectly legal. This means that we can avoid the modal "start link / end link" link creation style of many hypertext interfaces. In DHM's user interface, links can be created in two ways: (1) a "New Link" operation creating a link having one endpoint based on the current selection in the active editor (Figure 3); in this case no instantiation or link editor is opened. And, (2) via a new node operation creating a link with an open instantiation and link editor; in this case the link has no endpoints (see the 'Link 78' link instantiation in Figure 4). Endpoints can be added to the link at any time and as shown in Figure 4, links can have other links as an endpoints.

In our implementation of links, we confronted two problems with Dexter's model: 1) its aversion to dangling links and 2) its notion of link directionality.

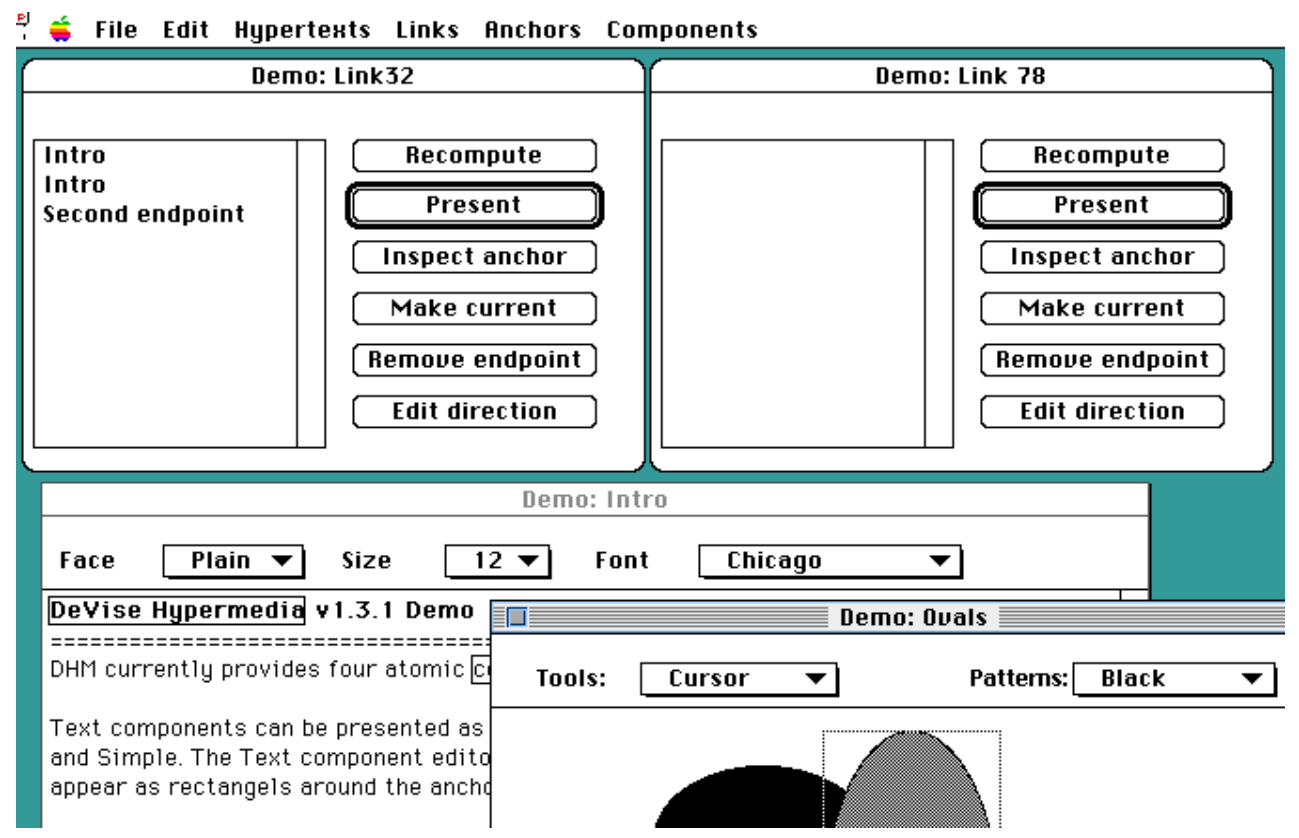

Figure 4: Two open link instantiations. Link32 has three endpoints, two in the 'Intro' text component, the third in the 'Ovals' component. Link 78 has no endpoints. 


\section{Dangling links}

In spite of Dexter's explicit aversion to dangling links, we chose to support them for several reasons. First, they allow lazy updating and garbage collection following node and anchor deletion. This is useful when the link to be deleted (or modified) lives on another machine or is currently locked by another user. A second, related situation involves data objects outside the control of the hypermedia, for example, files with component data needing to be moved or deleted. Third, the dangling endpoint can be "re-linked" or re-connected to another node or anchor without having to rebuild the entire link (especially useful for multi-headed links). Finally, dangling links can be created intentionally as place holders when the desired endpoint node or anchor does not yet exist. ${ }^{2}$ The presence of such dangling links could be monitored by the system either on command or automatically. Users could then be prompted to reconnect "missing" link endpoints.

We imagine four different dangling link situations arising in an integrated Dexter-based hypermedia system: 1) the endpoint's component has been deleted, 2) its anchor has been deleted, 3) relevant data objects referred to by the component's contents are unavailable, and 4) the anchor value is invalid. In the first two cases, the deletion operation modifies the objects so that later calls to followLink raise exceptions. Component deletion is implemented by clearing the anchors list and component contents, and setting a "deleted" flag. Anchor deletion is carried out similarly.

Cases 3 and 4 usually result from actions outside the control of the hypermedia. For example, data objects making up a component's contents can become unavailable if the contents is a file identifier, and the file has been moved or deleted independent of the hypermedia (case 3 ). In this case the followLink operation should catch the file system exception and pass it along as a dangling exception to the user.

In case 4, the data specified by the anchor value becomes invalid when relevant parts of the component's contents are modified with editors outside the hypermedia. This situation is impossible to detect in general during a CreateLinkMarker or a FollowLink operation, since the lookup/computation of anchor value may not raise an exception. An example is when the anchor value is still legal but out of date, as a result of "unauthorized" editing of the surrounding text. Currently in DHM, we have implemented detection and re-link options for case 1.

\section{Link directionality}

The Dexter model includes only minimal motivation for its notion of link directionality. We are told that each link specifier indicates a directionality using one of the constants FROM, TO, BIDIRECT, or NONE, depending on whether the endpoint is to be interpreted as a source, destination, both source and destination or neither, respectively. Furthermore, every link must have at least one TO specifier. ${ }^{3}$ Such directionality constants are used to model the link semantics of existing hypermedia systems. For example, Intermedia links are modeled with BIDIRECT directionality on all specifiers. This is because the endpoints of an Intermedia link are directionally interchangeable [6]. ${ }^{4}$ In NoteCards, on the other hand, links have a definite source and destination [9].

However, this scheme seems insufficient to model the ways people interpret link direction in

\footnotetext{
${ }^{2}$ An anonymous reviewer mentioned an example from asynchronous collaborative writing: When sharing parts of a hypertext, the links should dangle while being shared, but be re-attached when returned.

${ }^{3} \mathrm{An}$ anonymous reviewer informs us that the wording of the constraint should have been, "at least one TO or BIDIRECT specifier."

${ }^{4}$ Intermedia anchor attributes can, however, be notated with directionality information.
} 
practice. Consider the following three notions of directionality:

Semantic direction: This concerns the semantic relationship between the components captured by the link. For example, a "Supports" link connecting components A and B has a direction in which it normally "reads"; the argument in Component A "supports" the claim in Component B [17, Ch. 4].

Creation direction: This direction corresponds to the order in which the link endpoints were created: the source of the link is the first endpoint created while the destination is the last.

Traversal direction: This direction specifies how the link can be traversed. HyperCard links, for example, can only be traversed from source to destination. ${ }^{5}$ NoteCards links can be traversed in both directions, although the interface style is different. When moving from source to destination, one clicks on the source anchor's icon. To move from destination to source, a menu of "back-links" is opened in the destination component and the appropriate link icon is chosen.

These senses of link direction are in principle orthogonal. For example, the directions in which one can physically traverse a link in a particular system need not depend on the link's semantic direction. Nonetheless, many systems enforce dependencies. In NoteCards, for example, the creation direction corresponds to the traversal direction.

DHM currently supports selection of creation and traversal directions in the user interface, based in part on the Dexter proposal for direction attributes on link specifiers. When creating a link the user can choose the first endpoint to be either Source, Destination or Both (see Figure 3 ). This corresponds to setting either FROM, TO or BIDIRECT, respectively, as the value of the direction attribute on the corresponding specifier. Similar choices are available when adding endpoints to a link with the Add Endpoint operation. By default, endpoints created using New Link are Sources and those created with Add Endpoint are destinations.

The direction values recorded in the specifiers support the user's choice of traversal direction. As shown in Figure 5, the Follow Link operation can be invoked with a direction parameter.

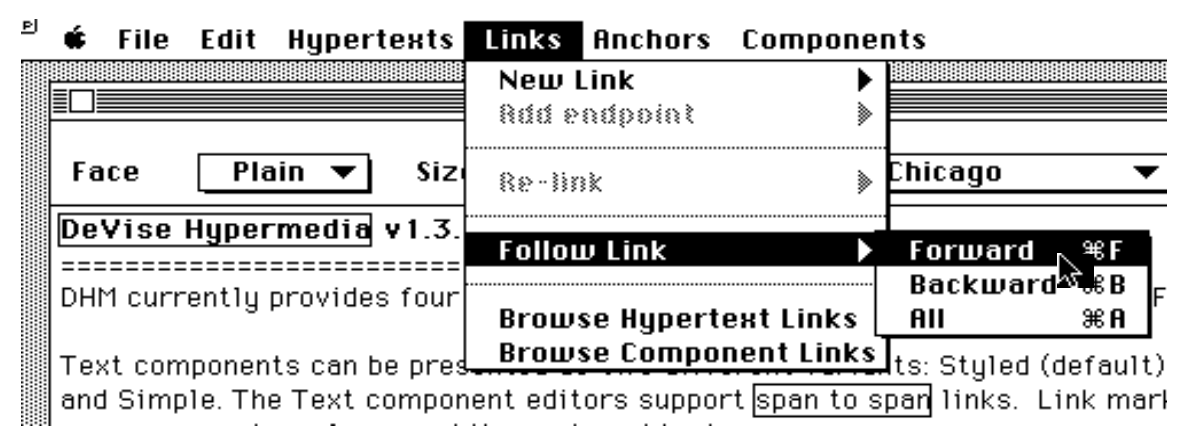

Figure 5: FollowLink invoked with a direction parameter.

When following a link in the Forward direction, endpoints with Direction value TO or BIDIRECT are presented. In the Backward direction, endpoints with Direction value FROM or BIDIRECT are presented. When following a link in All directions, all endpoints are presented.

As described above, the Dexter direction values were originally introduced to model directionality in existing systems. Designers of systems based on the model need to make operational interpretations of the values. This is straightforward for the TO and FROM values, though less clear for NONE and BIDIRECT. In DHM we use BIDIRECT for endpoints that are

\footnotetext{
${ }^{5}$ This is because HyperCard links are implemented as "Go" statements in a script in the link's source component. This also means that link's cannot normally be seen from their destination cards.
} 
conceived of as both Source and Destination. Currently, we have not chosen a semantics for the NONE value, but it could be used to mark endpoints that (temporarily) should not be presented when following a link. Such endpoints would still be accessible, however, through a link instantiation (see Figure 4). Link instantiations also allow the user to change the direction values of the individual endpoints.

Semantic direction is not explicitly supported in DHM (nor in Dexter), but the general attribute mechanism that allows creation of different link types could also support assigning semantic directions to links.

\section{Anchors}

One of Dexter's major contributions is its explicit identification of anchors as the "glue" connecting network structures to the contents of particular components. Anchors are a controlled means of referring into the "within-component" layer. Without them, links connect only whole components.

Dexter's anchors are defined relative to a component and have an ID that is unique within that component. Link specifiers must identify both the component id and the anchor id. Explicit mention of the ids can be avoided, however, by use of the resolver function. Thus the component appearing at a link's endpoint can be computed dynamically at run-time.

The biggest problem with Dexter's model of anchors is that they are not properly related to composites. That is, although the contents of a composite (a list of baseComponents) is "visible" (i.e. explicitly represented) in Dexter, no mention is made of how anchors should refer to baseComponents within a parent composite. In DHM we allow composites to include fullfledged components (see Section 5), adding further problems. For example, can an anchor in the parent composite be tied to an anchor in one of its components? That is, can a link "indirect" through a composite's anchor, to an enclosed component's anchor?

There are other anchor-related issues not discussed in the Dexter model. Consider, for example, links to whole components. Should they have an empty Anchor reference in the specifier or should there be a "whole-component" anchor type? In that case, should all wholecomponent links share a single whole-component anchor, or should there be one anchor for each link endpoint? Indeed the general issue of sharing versus multiplying anchors is left open in Dexter. When creating a new link, should one always try to reuse any existing applicable anchor? Suppose there is more than one?

DHM extends Dexter's model of anchors in several ways. First, we use dynamic references ("pointers") instead of anchor ids. ${ }^{6}$ This means that link specifiers point directly at component anchors avoiding the need for an accessor function. Similar benefits accrue from our blockstructured type definitions. For example, a component need not include an explicit reference to its enclosing hypertext, since the component definition is nested within the definition of a hypertext. Likewise, an anchor need not include an explicit reference to its enclosing component.

DHM distinguishes three high-level anchor types which are independent of the type of the enclosing component. Whole-component anchors support the degenerate case of link endpoints not anchored within a component's contents. ${ }^{7}$

A marked anchor is one for which an object is directly embedded in the component's contents.

\footnotetext{
${ }^{6}$ Utilizing an OODB makes our pointers persistent. We nonetheless maintain component and anchor ids in order to be able to generate transportable interchange formats for the hypermedia structure.

${ }^{7}$ In DHM, all links with whole-component endpoints in a component share a single whole-component anchor.
} 
This object is called a link marker in Dexter. It may or may not be visible - indeed, some link markers (e.g. an Emacs "mark") may never be made visible as such. Link markers can be implemented in a variety of ways depending on the medium and the application. Visible icons inserted in text or graphic windows can serve as link markers (e.g. NoteCards link icons). But a link marker can also correspond to what Meyrowitz [14] calls a "permanent tie." Such an object can "track" editing changes to the component's contents including changes to the selection itself. The instantiation may or may not choose to make the link marker visible (see e.g. Intermedia's arrow icon registering the presence of a permanent selection). DHM supports link markers in text components by maintaining outlined regions around the anchored text selections (see e.g. Figure 5). A command-click within the link marker region invokes a follow on the corresponding marked anchor's links.

Unmarked anchors have no link markers; normally their location within a component must be computed. Text components in DHM support a particular kind of unmarked anchor called keyword anchor, resembling the endpoints of HyperTies text links [16]. As an example, consider the situation of creating a new link shown in Figure 3. Assume that creating this new link requires creating a new anchor (as opposed to reusing an existing one), and that the anchor is to be a keyword anchor. In that case, a copy of the selected text string "components" is saved as the anchor's value. If we later invoke FollowLink from this component (assuming we haven't selected some marked anchor), the values of all keyword anchors will be checked against the currently selected text. In particular, if the current selection's text matches the string "components", then the link created earlier in Figure 3 will be followed.

What sets a marked anchor apart from an unmarked one is the ability to retrieve the anchor directly from a selection in the component's editor. If a link marker is currently selected (or clicked on) in an active instantiation, then the instantiation is able to directly access the corresponding marked anchor. This is in contrast to unmarked anchors, where a search is required. In general, each unmarked anchor must be asked whether it is currently "selected" (or perhaps more descriptively, "applicable"). The operation of following a link from a marked anchor takes constant time, whereas following a link from an unmarked anchor requires in the worst case time proportional to the total number of unmarked anchors in the component. ${ }^{8}$

\section{Structures}

The notion of structure (usually hierarchical) has been a part of most hypertext systems since the time of NLS/Augment in the 60's [2]. For example, in KMS (as well as its ancestor ZOG), a hierarchical structuring capability is built in to every node [1]. That is, all nodes (called "frames" in ZOG/KMS) can act as containers for other nodes. Usually, however, hierarchical structuring (and on rare occasions, non-hierarchical structuring), is supported through separate mechanisms.

In his landmark "Seven Issues" paper [7], Halasz proposed that the composite be elevated to peer status with atomic nodes and links. Composites would provide a means of capturing nonlink based organizations of information, making structuring beyond pure networks an explicit part of hypertext functionality. ${ }^{9}$ Halasz also argued for the related notions of computed and virtual composites. The contents of a computed composite might be, say, the result of a structural query over the hypertext returning sets of nodes and links as "hits." A virtual composite is created on demand at runtime, but not saved in the database. Later, in Aquanet [13], the composite idea was used to capture slot-based structures consisting of nodes and relations, multi-

\footnotetext{
${ }^{8}$ This can be improved using hash tables and the like.

${ }^{9} \mathrm{~A}$ similar appeal was made by van Dam in his attack on links as "go to" statements [19].
} 
headed variants of links.

Halasz [7] also criticized purely link-based structures arguing that they lack a single node capturing the overall structure. The Dexter model's composite addresses this critique. As an aggregation of base components, it acts both as a full-fledged node in the network, and as container for the structure. In particular, such a composite can contain link components (in addition to atomic nodes and other composites) and thus capture complex non-hierarchical network structures (like Aquanet relations). Furthermore, because of Dexter's clean separation of storage and runtime environments, virtual composites are a simple variant.

Though Dexter's notion of composite is a significant step forward, it is only one point in a spectrum of possible designs, each having certain advantages and meeting certain needs. Our architecture extends some of the features of composites to all components and supports tailoring for particular applications. Users adding a new component type to our prototype make choices along several dimensions:

\section{Virtual / non-virtual components}

Any component type (not just composites) can be made virtual by setting a flag. Such components resemble normal components, but are only saved in the databse if pointed at by another component (say, a link). Virtual components resemble objects in a dynamic programming environment; if they are not pointed at, then garbage collection reclaims them. For example, a virtual component might be created automatically to display the results of a userinstigated search over the components in the hypertext. Such a component persists beyond the current session only if the user creates a link to (or from) it, or adds it to an existing non-virtual composite.

\section{Computed / static components}

Any component type (again, not just composites) can be the result of a computation rather than manually created by the user. A typical example is a component created on the basis of executing a query. An attribute contains the information used to perform the computation. The component's contents can later be recomputed, either on demand or automatically. Some computed components (like browsers) reflect the contents or structure of parts of the network. In such cases, recomputation can be based on periodic checks of the relevant sub-net, or be forced by changes to the relevant components or structures.

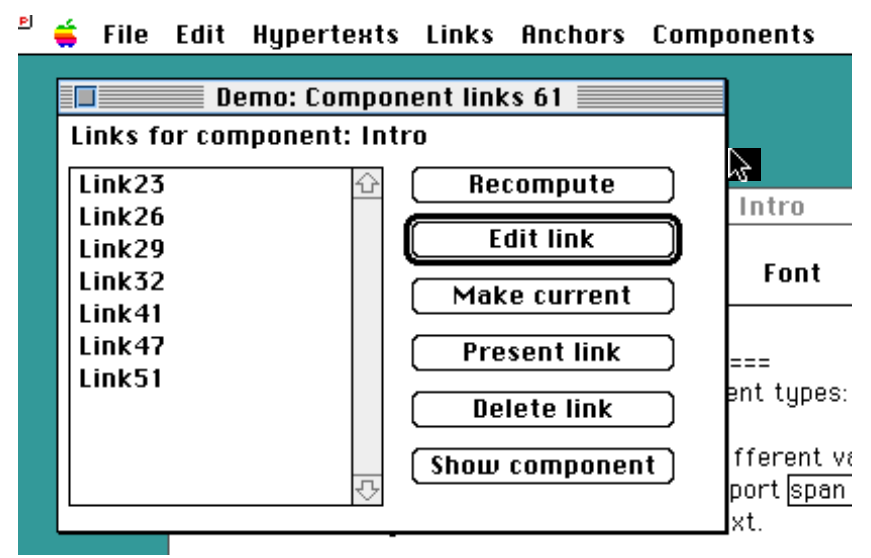

Figure 6: A link browser composite in DHM; lists all links to and from the 'Introduction' node.

\section{Component contents}

Typically, the contents of a component in a hypermedia system is not simply a flat set of 
enclosed data objects as suggested by the Dexter model. The contents are often structured and can include external data objects or references to other components

Figure 6 shows an example of a composite type supporting link browsing in DHM. The Link browsers are implemented as virtual, computed composites with contents consisting of lists of references to LinkComponents. Though not anticipated by the Dexter model, this kind of component was fairly easy to implement using the framework described above.

\section{Integration and component contents}

The phenomenon of system developers "owning the world" is becoming increasingly rare. Today, most practical computer environments consist of several third-party applications, perhaps customized for particular work settings by local programmers or user "tailors." Unfortunately, the application's inner workings and structures are rarely open to the developer trying to integrate them into a larger environment. The problem is exacerbated if the environment includes a variety of platforms.

In the last few years, researchers and developers have tried to use hypermedia to address this integration problem $[6,11,14,15]$. They argue that rather than build a hypermedia system that includes all the applications needed in the work setting, one should employ hypermedia as a linking architecture, "connecting" the world rather than "owning" it.

The Dexter reference model makes certain important contributions to this effort. At the architectural level, Dexter distinguishes between objects belonging to the hypermedia (both runtime and storage), and the "within-component layer" belonging to an application. In addition to describing the hypermedia data model, Dexter offers two important concepts that help cross the boundary: anchors and presentation specifications (or "pspecs"). Anchors support linking to and from points within the contents of an application document. Pspecs provide a means of storing with a Dexter component information on how to start and configure the appropriate application.

In this way, Dexter opens the possibility of integrating third-party applications into a linked hypermedia environment. But it leaves unaddressed at least two important integration-related questions. First, Dexter does not distinguish between components whose contents are managed (in particular, stored) by the hypermedia and those whose contents are managed by third-party applications.

The second problem involves application documents having internal structure. Such documents can be integrated as a single unit into the hypermedia using a component "wrapper," but often the document's internal structure needs to be "exposed" for link anchoring. Dexter suggests using composite components, but says almost nothing about how to anchor within the subcomponents of a composite. Nor does it discuss whether or how a composite component's structure should model the internal structure of an application document.

In what follows, we discuss various possibilities for storing and structuring component contents.

\section{Atomic components}

Figure 7 shows two possible relations between an atomic component and an anchored data object. 


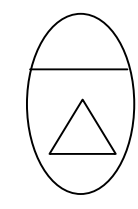

(a)

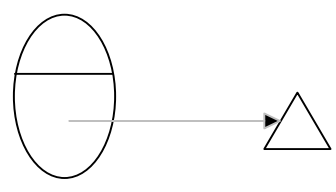

(b)

Figure 7: Data is either part of an atomic component's contents or referenced by it. Dotted arrows denote references out of the hypermedia structure, e.g. file identifiers.

Figure 7a shows the traditional situation where an application and its data objects are built into the hypermedia system. DrawComponents in DHM, for example, encapsulate lists of graphical objects stored in the OODB together with the components.

In Figure $7 \mathrm{~b}$, on the other hand, data objects wrapped by a component are stored separately and only referenced by the contents of the component. In DHM such a component/data object relationship characterizes FileComponents and MovieComponents. FileComponents are used to wrap arbitrary files in the file system, using file ids stored in the component contents. In this way, DHM supports linking (using WholeComponent anchors) to documents created with applications like Microsoft Word or Excel. The followLink operation automatically launches the appropriate applications on the files.

MovieComponents "wrap" Quicktime movies, ${ }^{10}$ large multimedia data objects (from five to several hundred MB) too complex to be easily stored in the hypermedia's OODB. Hence, they are better handled using MovieFiles referred to by the component contents. ${ }^{11}$ In this case, the component contents is again a file identification object.

Typically, an atomic data object belongs to exactly one atomic component. But there are cases where two or more components need to share data. Here the components could have different types and/or different sets of anchors. Such multiple "views" can be supported by the containment style shown in Figure 7b. An example is movie files that are shared across several hypertexts.

\section{Composite components}

With regard to more complex structures of components and data objects, we found Dexter's notion of composite too narrow. According to Dexter, a composite may only contain encapsulated data objects (see for example, the bottom left composite in Figure 8). As noted by Halasz \& Schwartz [8] this kind of composite can model structures like graphical canvases. For other applications, however, composites need to refer to external data objects or other components. In the following we discuss examples of such composite types.

\section{Composites "containing" components}

We first consider composites that refer to other components as shown in Figure 8. One example is the TableTopComposite used to save configurations of components presented together on the screen [18].

\footnotetext{
${ }^{10}$ QuickTime ${ }^{\mathrm{TM}}$ by Apple Computer Inc. implements a format for storing/compressing digitized video.

${ }^{11}$ In the current version of DHM, we support only one movie per MovieFile (and thus, one per component).
} 


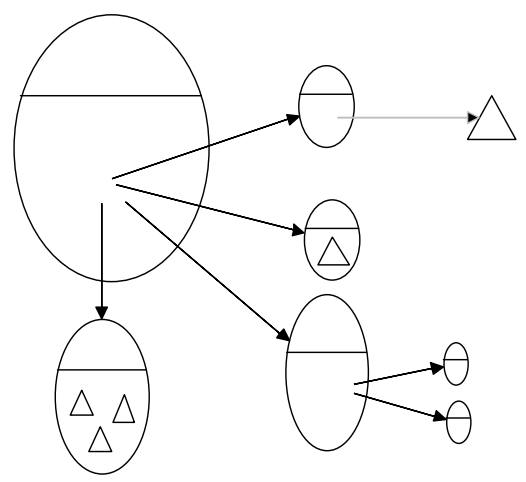

Figure 8: A composite referring to components of arbitrary type. Solid arrows denote internal pointers to hypermedia components.

The contents of a TableTopComposite in DHM is a list of "pointers" to components of arbitrary type (including links and other composites); the composite does not directly contain or wrap the data objects.

Another example is a search composite. Here the contents is a list of components (again of arbitrary type) resulting from a title search or a query over component attributes. In DHM, such search composites are implemented as virtuals (see Section 5).

Figure 9 shows a slightly different kind of composite also used to group components. In this case, the composite is both virtual and has contents restricted to certain component types.

Figure 9: A virtual composite restricted to refer to LinkComponents. Shading indicates that the composite is virtual.

The VirtualLinkComposite shown in Figure 9 is used in DHM to implement a variety of link browsers. VirtualLinkComposites are "computed" composites; their creation requires collecting a set of links for an entire hypertext, a specific component, or a specific anchor, depending on the kind of link browser.

When appropriate, restricting the component types pointed at by a composite allows customization of the composite's interface. For example, the VirtualLinkComposite interface supports inspecting individual link specifiers. A non-typed composite would require runtime checking of the types of contained objects.

Encapsulated data objects

Up to this point we have focused on composites referring to other components; we now turn to composites referring directly to data objects. In Figure 10, the data objects depicted as triangles are encapsulated in a "container" object (drawn as a rectangle). In this case, the internal structure 
of the rectangular object is visible to the hypermedia system. Hence the composite and its nested components can refer both to the enclosing object and to its internal structure. ${ }^{12}$

${ }^{12} \mathrm{~A}$ nested component is one whose definition lies within the block structure of the parent component and thus can only be seen in the context of the parent component. (The block structure of DHM's object-oriented component definitions is similar to the block structure found in procedural programming languages like Pascal.) 


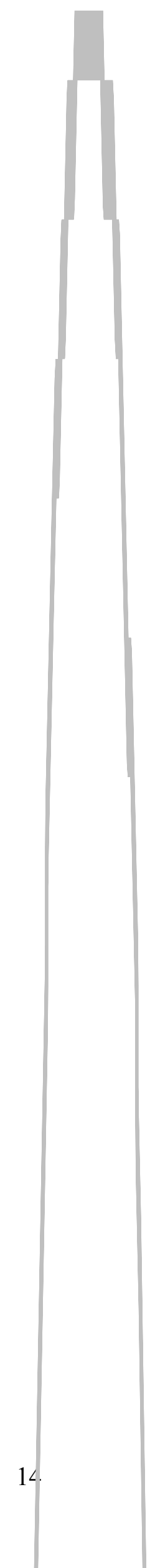


Figure 10: Typed composite with nested components points at encapsulated data objects.

An example of such a composite is used to represent modules in the Mjølner Beta programming environment [12]. Mjølner supports fine grained modularization of programs using atomic modules called 'fragments' contained in parent 'fragment groups'. Each fragment group is stored on a file. To represent such structures in DHM, we use a FragmentGroupComposite whose contents includes a reference to a fragment group file and a list of references to atomic FragmentComponents, declared inside the block structure of the FragmentGroupComposite. The nested structure of the 'real world' data objects (fragments and fragment groups) is mapped directly onto the nested structure of the representing components. Hence, we can link both to the composite and to the nested atomic components representing individual fragments.

We provide anchors at the FragmentGroupComposite level, to comments made at the group level, and at the FragmentComponent level, to comments and source code belonging to individual fragments.

Structured composites

An Aquanet relation [13], is an example of a hypermedia composite with structured contents. A fundamental feature of an Aquanet relation is that it resembles a multi-headed link with named endpoints.

We suggest implementing such relations as composites with contents consisting of a keyed table of component references (see Figure 11). Such a composite can refer to basic objects (atomic components) as well as to other relations (structured composite components). In addition, instantiations of such composites can support link-like "endpoint" presentation. Here, "endpoints" refer to the components pointed at by the composite's encapsulated structure. 


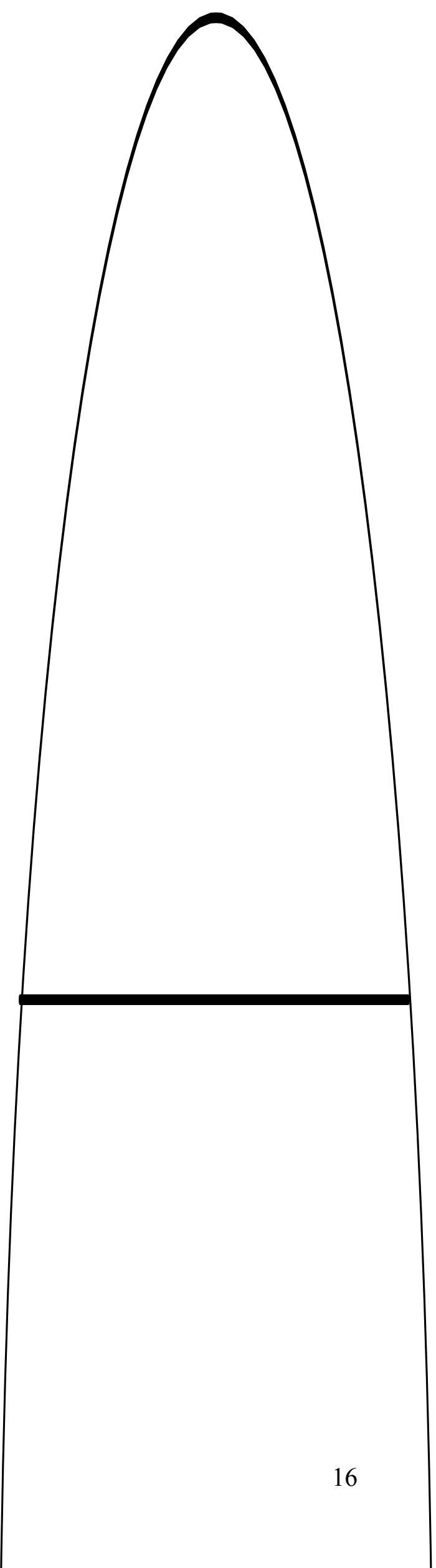


Figure 11: A composite with structured contents.

\section{Summary}

The above examples show the need to support a broad view of component contents when developing open Dexter-based hypermedia systems. Integrating components of the Storage layer with data objects of the within-component layer is one important aspect (as illustrated by the difference between a DrawComponent and a MovieComponent). Another is the internal integration of components and composites as illustrated by the cases of TableTopComposite and VirtualLinkComposite. Table 1 summarizes the discussion in this section along three dimensions.

\begin{tabular}{|l|l|l|}
\hline Structure of contents & Type/location of contents & Definition of contents \\
\hline - Atomic & - Data objects & $\bullet$ Encapsulated in this \\
- Unstructured collection & - within component & component \\
- Structured collection & - outside component & $\bullet$ \\
- sorted list & - Components & \\
- keyed table & - restricted types & \\
- tree & - unrestricted & \\
- ... & & \\
\hline
\end{tabular}

Table 1: Three aspects of component contents.

\section{Concluding remarks}

This paper discussed experiences from Dexter-based hypermedia development in the DeVise project at Aarhus University. The work has lead to clarifications and extensions to the Dexter model. Those treated in this paper are concerned with integration issues and the design of central object classes like links, anchors, and composites.

Our work on Dexter based hypermedia contributes to the Esprit III project, EuroCODE, aimed at developing a CSCW Open Development Environment, a so-called "CSCW shell." One of the EuroCODE activities involves extending our Dexter based hypermedia architecture to support cooperation via long term transactions, flexible locking and event notifications. The open, extensible architecture we are developing comprises an object oriented framework for developing multi-user hypermedia applications [3].

\section{Acknowledgments}

This work has been supported by the Danish Research Programme for Informatics, grant number 5.26.18.19. Our thanks also go to the members of the DeVise project at Aarhus University and four anonymous reviewers.

\section{References}

1. Akscyn, R., McCracken D., \& Yoder, E. 1988. KMS: A distributed hypermedia system for managing knowledge in organizations. CACM, 31, 7, (July), 820-835. 
2. Engelbart, D. C. 1984. Authorship provisions in AUGMENT. Proceedings of the 1984 COMPCON Conference, COMPCON '84 Digest, (San Francisco, Calif., February), pp. 465472.

3. Grønbæk, K, Hem, J.A., Madsen, O.L., \& Sloth, L. Designing Dexter-Based Cooperative Hypermedia. In this volume.

4. Grønbæk, K. \& Knudsen, J. L. Tools and Techniques for Experimental System Development. In Systä, K., Kellomäki, P., \& Mäkinen, R.(eds.) Proceedings of the Nordic Workshop on Programming Environment Research, Tampere, Finland, January 8-10, 1992.

5. Grønbæk, K., Kyng, M., \& Mogensen, P. CSCW challenges in large-scale technical projects - a case study. To appear in Proceedings of Conference on Computer Supported Cooperative Work '92, Toronto, Ontario, November, 1992.

6. Haan, B.J., Kahn, P., Riley, V.A., Coombs, J.H., \& Meyrowitz, N.K. IRIS Hypermedia Services. Communications of the ACM 35(1), January 1992, pp.36-51.

7. Halasz, F. 1988. Reflections on NoteCards: Seven issues for the next generation of hypermedia systems. Commun. ACM, 31, 7, (July), 836-852.

8. Halasz, F., \& Schwartz, M. 1990. The Dexter hypertext reference. Proceedings of the Hypertext Standardization Workshop, (Gaithersburg, Md., January), pp. 95-133.

9. Halasz, F., Moran, T., \& Trigg, R. 1987. NoteCards in a nutshell. Proceedings of the CHI '87 Conference, (Toronto, Canada, April), pp. 45-52.

10. Hem, J.A., Madsen, O.L., Møller, K.J., Nørgaard, C., \& Sloth, L. Object Oriented Database Interface. Deliverable D5.2, ESPRIT project 5305 EuroCoOp IT Support for Distributed Cooperative Work, December 1991.

11. Kacmar, C.J. \& Leggett, J.J. PROXHY: A Process-Oriented Extensible Hypertext Architecture. ACM Transactions on Information Systems 9(4), October 1991, pp. 399-419.

12. Kristensen, B.B., Madsen, O.L., Møller-Pedersen, B., \& Nygaard, K: Object-Oriented Programming in the Beta Programming Language. Addison-Wesley, forthcoming (1992).

13. Marshall, C.C., Halasz, F.G., Rogers, R.A., \& Janssen, W.C. Aquanet: a hypertext tool to hold your knowledge in place. Proceedings of Hypertext '91, ACM New York, December 1991, pp. 261-275.

14. Meyrowitz, N. The Missing Link: Why We're All Doing Hypertext Wrong. In Barrett (ed.) The Society of Text. MIT Press, Cambridge Massachusetts, 1989, pp. 107-114.

15. Pearl, Amy. 1989. Sun's link service: A protocol for open linking. Proceedings of the Hypertext '89 Conference, (Pittsburgh, Pa., November), pp. 137-146.

16. Shneiderman, B. 1987. User interface design for the HyperTIES electronic encyclopedia. Proceedings of the Hypertext '87 Conference, (Chapel Hill, November), pp. 189-194.

17. Trigg, R. 1983. A network-based approach to text handling for the online scientific community. Ph.D. dissertation. University of Maryland (University MicroFilms \#8429934), College Park, Md. 
18. Trigg, Randall. 1988. Guided tours and tabletops: Tools for communicating in a hypertext environment. ACM Trans. Off. Inf. Syst., 6,4, (October), 398-414.

19. van Dam, A. 1988. Hypertext '87: Keynote Address. CACM, 31, 7, (July), 887-895.

CR Categories and Subject Descriptors: E.1 [Data Structures]: Hypertext; H.1.2 [Models and Principles]: User/Machine Systems - human information processing; H.2.1 [Database Management]: Logical Design - hypertext; H.3.2 [Information storage and retrieval]: Information storage - hypertext; H.4.2 [Information Systems Applications]: Types of Systems -hypermedia; H.5.1 [Multimedia Information Systems]: Hypertext Navigation and Maps; I.7.2 [Document Preparation]: Hypertext/Hypermedia.

General Terms: Hypermedia, hypertext.

Additional Key Words and Phrases: Dexter model, Composites, Dangling Links, Open Hypermedia.

\section{ABOUT THE AUTHORS:}

KAJ GRØNB/EK is assistant professor at the Computer Science Department, Aarhus University, Denmark. Current research interests include cooperative design, Computer Supported Cooperative Work, development and use of hypermedia technology, and system development with focus on object oriented tools and techniques. Authors' Present Address: Computer Science Department, Aarhus University, Bld. 540, Ny Munkegade; DK-8000 Aarhus C; Denmark; email: kgronbak@daimi.aau.dk.

RANDALL H. TRIGG is a member of the research staff at Xerox Palo Alto Research Center. Current research interests include participatory design, the design and evolution of tailorable computer systems, hypermedia, computer supported cooperative work, and connections between social science and system design. Authors' Present Address: Xerox Palo Alto Research Center, 3333 Coyote Hill Road, Palo Alto, CA 94304; email: trigg@parc.xerox.com. 